\section{Consumo de álcool e risco para doença coronariana na região metropolitana de São Paulo: uma análise do Projeto GENACIS}

\section{Alcohol consumption pattern and Coronary Heart Disease risk in Metropolitan São Paulo: analyses of GENACIS Project}

\section{Maria Cristina Pereira Lima' \\ Florence Kerr-Côrrea' \\ Jurgen Rehm"}

' Faculdade de Medicina da Universidade Estadual Paulista "Júlio de Mesquita Filho" - UNESP, Botucatu, SP.

"Social and Epidemiological Research (SER) Department, Centre for Addiction and Mental Health, Toronto, Canada; Population Health Research Group, $\mathrm{CAMH}_{\text {, }}$ Canada; Addiction Policy, Dalla Lana School of Public Health, University of Toronto (UofT), Canada; Department of Psychiatry, Faculty of Medicine, UofT, Canada; PAHO/WHO Collaborating Centre on Mental Health \& Addiction; Epidemiological Research Unit, Technische Universität Dresden, Klinische Psychologie \& Psychotherapie, Dresden, Germany; Graduate Department of Community Health and Institute of Medical Science, UofT.

O trabalho se refere a analise de dados do projeto GENACIS realizado em São Paulo. O presente estudo foi desenvolvido em estagio pós-doutoral no Centre for Addcition and Mental Health na Universidade de Toronto, Canadá (Coordenaçao de Aperfeicoamento de Pessoal de Nivel Superior, CAPES, Processo BEX3552/07-1)

Correspondência: Maria Cristina Pereira Lima. Departamento de Neurologia e Psiquiatria, Faculdade de Medicina de Botucatu, Universidade Estadual Paulista Júlio de Mesquita Filho, Distrito de Rubião Jr., Botucatu, SP, Brazil, 18618-000. E-mail: kika.botucatu@gmail.com

\section{Resumo}

Objetivos: Examinar a associação entre consumo de álcool e risco para doença coronariana em amostra populacional. Métodos: Estudo transversal, de base populacional, conduzido de janeiro/2006 a junho/2007, na região metropolitana de São Paulo, como parte do estudo internacional (Gender, Alcohol, and Culture: an International Study). Os sujeitos (1.501, sendo 609 homens e 892 mulheres) eram residentes da região metropolitana de São Paulo, tinham 30 anos ou mais de idade e foram selecionados aleatoriamente, a partir de amostragem complexa por conglomerados. Todos os indivíduos consentiram em participar da pesquisa. A variável dependente foi risco cardíaco avaliado através do WHO Rose Angina Questionnaire. A análise multivariada consistiu em regressão logística, tendo sido realizado ajuste para uso de tabaco e índice de massa corpórea. Resultados: A taxa de resposta foi $75 \%$. Ser mulher, ter mais idade, ser negro, fumante e ter um índice de massa corpórea elevado, foram associados a maior risco para doença coronariana. Indivíduos que nunca beberam na vida ( $\mathrm{OR}=2,22$ ) e ex-bebedores ( $\mathrm{OR}$ $=2,42$ ) tiveram maior risco de doença cardíaca do que aqueles que informaram beber até $19 \mathrm{~g}$ de álcool por dia, sem episódios de beber excessivo. Entre os que tiveram episódios de embriaguês observou-se uma tendência a maior risco ( $O R=3,95, p=0,09)$. Conclusões: Nossos achados sugerem um menor risco para doença coronariana entre os bebedores moderados. Destaca-se que os estudos que avaliam o impacto do álcool sobre doença cardíaca precisam identificar o padrão de uso de álcool dos sujeitos, visto que este aspecto pode modificar o risco. Políticas públicas são necessárias para reduzir o uso nocivo de álcool e a morbidade a ele relacionada no país.

Palavras-chave: Álcool. Consumo de álcool. Doença coronariana. Estudo transversal. Epidemiologia. 


\section{Abstract}

Objectives: To examine the association between patterns of drinking and coronary heart disease (CHD) risk in a populational sample. Methods: A population-based cross-sectional study carried out from January 2006 to June 2007, in Metropolitan São Paulo, Brazil, in conjunction with the international collaborative GENACIS project (Gender, Alcohol, and Culture: an International Study), with PAHO support. The subjects (1,501; 609 men, 892 women) of this study were residents of randomly chosen households aged 30 years and above who consented to provide information. The dependent variable was cardiac risk as assessed by the WHO Rose Angina Questionnaire. Logistic Regression analysis was used and the data were adjusted for Body Mass Index (BMI) and smoking. Results: The response rate was $75 \%$. Being female, older, African-American, a current smoker, and having a greater BMI were associated with higher risk of coronary heart disease. Lifetime abstainers $(\mathrm{OR}=2.22)$ and former drinkers $(\mathrm{OR}=2.42)$ had greater CHD risk than those who consumed up to 19g pure alcohol per day, with no binge. Among those who had binged weekly or more there was a tendency toward higher risk (OR $=3.95, \mathrm{p}=.09$ ). Conclusions: Our findings suggest a lower risk for $\mathrm{CHD}$ among moderate drinkers. It is important, in studies which were investigating cardiac risk, assess heavy alcohol use, since it can change that risk. Also, effective public policies are needed to reduce harmful drinking and related morbidity in Brazil.

Keywords: Alcohol. Alcohol consumption. Coronary heart disease. Cross-sectional study. Epidemiology.

\section{Introdução}

Estudos epidemiológicos têm mostrado que o consumo de álcool é um importante fator de risco para inúmeras doenças, bem como para acidentes de trânsito e mortes por causas violentas ${ }^{1,2}$. Sua relação com doenças vasculares, em especial com doença coronariana, mantém-se, no entanto, um tema controverso. Embora haja evidências de efeitos benéficos do uso moderado das bebidas alcoólicas ${ }^{3}$, há estudos apontando um maior risco de doença coronariana associado ao padrão de uso do álcool* do tipo beber excessivo ${ }^{4-6}$. Ingerir grandes quantidades de álcool em uma única ocasião tem sido associado com efeitos adversos sobre a pressão arterial e também sobre a agregação plaquetária. McKee \& Britton ${ }^{4}$ observaram que bebedores "pesados" haviam adquirido alterações nas lipoproteínas de baixa densidade, sem apresentar os efeitos cardioprotetores nas lipoproteínas de alta densidade. O beber "pesado" tem sido associado ainda a um aumento no risco de trombose ocorrendo após a parada da ingestão, tendo sido também encontrada uma predisposição a mudanças histológicas, tanto no músculo cardíaco quanto no sistema de condução ${ }^{4}$. Estes achados sugerem que o beber pesado possa preencher os critérios de causalidade para óbitos por doença coronariana ${ }^{7}$. Ao lado disto, observa-se que consumo de álcool maior ou igual a sete drinques por semana associa-se negativamente à procura por testes de rastreamento para fatores de risco para doença coronariana, como dosagens de colesterol ou triglicérides ${ }^{8}$.

Diante da tendência global de aumento na expectativa de vida, doenças coronarianas têm se tornado importantes desafios em países como o Brasil, no qual a maior parte da sobrecarga das doenças cardiovasculares está relacionada à doença coronariana ${ }^{9,10}$. Entretanto, poucos estudos examinaram a relação entre álcool e as doenças cardiovasculares em geral e

\footnotetext{
* Não ha um consenso na literatura sobre o termo mais adequado para denominar o padrão de uso de álcool considerado excessivo. No presente artigo o termo a ser utilizado "pesado" se refere à ingestão episódica ou habitual de cinco ou mais drinques em uma única ocasião, salvo quando mencionado critérios diferentes deste.
} 
as coronarianas, em especial. Entre estes, Moraes et al. ${ }^{11}$ encontraram uma associação positiva entre consumo de álcool (medido em gramas por dia) e a incidência de doença cardiovascular. Em outro estudo, Piegas et al. ${ }^{12}$ observaram que consumo de álcool foi fator de proteção contra infarto agudo do miocárdio. Infelizmente, estes estudos não identificaram padrão de ingestão de álcool, o que poderia explicar em parte os resultados aparentemente incongruentes ${ }^{11,12}$.

O objetivo deste estudo é examinar a associação entre provável doença coronariana e padrão de ingestão de álcool em adultos residentes na região metropolitana de São Paulo. Nossa hipótese é que há uma associação entre estes, havendo um maior risco associado à doença coronariana entre aqueles que fazem ingestão pesada de álcool.

\section{Método}

Este estudo é parte do Projeto GENACIS, um acrônimo para Gender, Alcohol, and Culture: an International Study. Trata-se de um estudo transversal conduzido na região metropolitana de São Paulo (RMSP), que compreende 39 municípios e a capital do Estado, com cerca de 19 milhões de habitantes à época do inquérito. Esta pesquisa foi parte de um estudo multicêntrico, com apoio da Organização Panamericana de Saúde, cujo objetivo mais amplo foi investigar uso de álcool e sua relação com gênero. Os dados foram colhidos de janeiro de 2006 a junho de 2007.

\section{Participantes}

Os sujeitos deste estudo são adultos com 30 anos ou mais, selecionados através de amostra complexa por conglomerados, sendo o primeiro estrato o setor censitário e o segundo estrato o domicílio. A amostra é representativa da população urbana não institucionalizada da região metropolitana de São Paulo. A escolha por sujeitos nesta faixa etária se deveu ao fato de que o risco de doença coronariana, associado a estilo de vida, é maior em sujeitos mais velhos.
A taxa de resposta foi $75 \%$ havendo maior recusa de homens do que de mulheres. A amostra consistiu de 1.489 pessoas com 30 anos ou mais de idade, sendo 609 homens e 892 mulheres. Para maiores detalhes sobre amostragem consultar Taylor et al. ${ }^{13}$.

\section{Procedimentos}

Todos os entrevistadores tinham experiência previa com inquéritos domiciliares e foram especialmente treinados para este estudo. Dez por cento das entrevistas foram checadas pelos supervisores para controle de qualidade. Cartas esclarecendo os objetivos do estudo foram enviadas àqueles que se recusaram a participar na primeira tentativa, fornecendo ainda os telefones de contato com os investigadores principais, com o intuito de reverter recusas. Foi disponibilizado ainda um website com informações sobre o projeto (www.viverbem. fmb.unesp.br/pesquisa).

\section{Instrumentos}

A variável dependente foi risco cardíaco, avaliada pelo WHO Rose Angina Questionnaire (WHO-RAQ), em sua forma abreviada $^{14}$. Este questionário consiste em três questões: 1. Você tem qualquer tipo de dor ou desconforto (mal estar) no peito? 2. Você tem dor no peito quando caminha normalmente (não rapidamente) em lugar plano? 3. Você tem dor no peito quando caminha numa subida? Risco cardíaco se define pela resposta "sim" à primeira pergunta e "sim" à segunda ou terceira. É importante destacar que não se trata de doença cardíaca, mas sim de risco, identificado em nível populacional, corroborado por estudos que têm mostrado uma associação consistente entre a classificação de risco obtida pelo WHO-RAQ e risco coronariano em ambos os $\operatorname{sexos}^{15,16}$. A principal variável explanatória foi padrão de uso de álcool, controlando-se para variáveis demográficas, índice de massa corpórea e tabagismo. O uso de álcool foi estimado a partir do relato dos sujeitos. O questionário GENACIS investiga 
a quantidade e frequência de diferentes bebidas em um dia típico, procurando minimizar o viés de resposta. A quantidade de álcool ingerida foi expressa em gramas de etanol, obtidos através do cálculo de quantidade, tipo de bebidas e teor alcoólico destas, sendo calculada a ingestão em um dia típico. Um drinque foi padronizado para $13 \mathrm{~g}$ de etanol. Os sujeitos foram classificados de acordo com a ingestão de álcool em: abstinentes (que referiam nunca ter ingerido bebida alcoólica), ex-bebedores (que não ingeriram bebidas alcoólicas no ultimo ano) e bebedores (que ingeriram álcool no ultimo ano). Além do padrão habitual, foi perguntado também a respeito de episódios de beber excessivo (EBE), definido como ingestão de cinco drinques ou mais em um único dia, não tendo sido possível utilizar ponto de corte diferenciado para homens e mulheres. Os bebedores, por sua vez, foram classificados em "aqueles que ingerem até 19g de álcool, sem EBE", "aqueles que ingerem até $19 \mathrm{~g}$ de álcool, com EBE" e "aqueles com ingestão de $20 \mathrm{~g}$ ou mais".

Uso de tabaco foi definido por fumar diariamente ou ocasionalmente. Aqueles que já fumaram no passado, mas não fumam mais foram definidos como ex-tabagistas. O índice de massa corpórea foi calculado dividindo-se o peso pela altura elevada ao quadrado, sendo ambas as informações obtidas a partir do relato do entrevistado.

\section{Análise estatística}

Regressão Logística foi utilizada para calcular odds ratio (OR) e intervalos de confiança ajustados, tendo risco cardíaco como variável dependente, como mencionado acima. Foram incluídos nos modelos multivariados: variáveis sociodemográficas, tabagismo e índice de massa corpórea. Foi também testada a interação entre etnia e uso de álcool (Wald test), e comparados os modelos através do Teste da Razão de Verossimilhança ${ }^{17}$. Todas as análises foram realizadas utilizando o Stata 10 (Stata Corporation, College Station, Texas), incorporando os pesos utilizados para desenho amostral.

O estudo foi aprovado pelo Comitê de Ética em Pesquisa da Faculdade de Medicina de Botucatu em 13 de setembro de 2004.

\section{Resultados}

As características da amostra e a prevalência de risco para doença coronariana estão apresentadas na Tabela 1. Com relação ao consumo de álcool, a maior parte é abstinente $(32,5 \%)$ seguida daqueles que consumem 19g de álcool por dia típico. Metade da amostra nunca fumou e 21,6\% são tabagistas.

A prevalência de risco cardíaco estimada pelo WHO-RAQ foi $7,9 \%$.

Os odds ratio para risco de doença coronariana, após controle para variáveis sociodemográficas, tabagismo e índice de massa corpóreo estão apresentados na Tabela 2. Maiores riscos foram encontrados para os sujeitos mais velhos, para mulheres, ex-bebedores e para abstinentes a vida toda. A tendência para maior risco entre os que consumem até $19 \mathrm{~g}$ por dia com EBE não se alterou com a inclusão de outras variáveis no modelo. Não houve interação significativa entre consumo de bebidas alcoólicas e tabagismo.

Com respeito a outras variáveis introduzidas no modelo, ser negro quando comparado a ser branco teve um maior risco para doença coronariana $(\mathrm{OR}=1,98)$. De modo similar, o risco foi maior para fumantes (OR $=1,93$ ) quando comparados àqueles que nunca fumaram.

\section{Discussão}

Em síntese, observou-se maior risco para doença coronariana entre sujeitos mais velhos, mulheres, ex-bebedores, abstinentes a vida toda, fumantes e negros. Houve uma tendência a maior risco entre os sujeitos com consumo até $19 \mathrm{~g}$ de álcool por dia, com EBE.

O possível efeito protetor entre aqueles 
Tabela 1 - Características sociodemográficas, uso de álcool, tabagismo e risco cardíaco em amostra da região metropolitana de São Paulo $(n=1.489)$.

Table 1 - Demographic characteristics, drinking pattern, smoking and cardiac risk of Metropolitan Sao Paulo sample $(n=1,489)$.

\begin{tabular}{|c|c|c|}
\hline & $\mathrm{n}$ & $\%$ ajustado \\
\hline \multicolumn{3}{|l|}{ Sexo } \\
\hline Homem & 601 & 46.2 \\
\hline Mulher & 888 & 53.8 \\
\hline \multicolumn{3}{|l|}{ Idade $\left(\right.$ anos) $^{2}$} \\
\hline $30-39$ & 448 & 34.1 \\
\hline $40-49$ & 385 & 31.2 \\
\hline $50-59$ & 253 & 17.5 \\
\hline 60 ou mais & 402 & 17.2 \\
\hline \multicolumn{3}{|l|}{ Grupo étnico } \\
\hline Branco & 877 & 59.4 \\
\hline Negro & 155 & 10.9 \\
\hline Mulato & 405 & 26.9 \\
\hline Outros & 48 & 2.8 \\
\hline \multicolumn{3}{|l|}{ Uso de álcool ${ }^{3}$} \\
\hline Abstinentes (vida toda) & 577 & 32.5 \\
\hline Até $19 \mathrm{~g}$ sem EBE ${ }^{4}$ & 462 & 32.4 \\
\hline Até $19 \mathrm{~g}$ com EBE & 17 & 1.6 \\
\hline $20 \mathrm{~g}$ ou mais & 92 & 7.5 \\
\hline Ex-bebedores & 341 & 26.0 \\
\hline \multicolumn{3}{|l|}{ Tabagismo } \\
\hline Nunca fumou & 786 & 50.6 \\
\hline Ex-tabagistas & 385 & 27.8 \\
\hline Tabagistas & 318 & 21.6 \\
\hline \multicolumn{3}{|l|}{ Risco Cardíaco 5} \\
\hline Não & 1355 & 92.1 \\
\hline Sim & 134 & 7.9 \\
\hline
\end{tabular}

${ }_{1}^{1}$ Percentual ajustado para desenho amostral. ${ }^{2}$ Sem informação sobre 1 sujeito. ${ }^{3} \mathrm{O}$ volume de consumo de álcool está expresso em gramas de álcool por dia. ${ }^{4} \mathrm{EBE}=$ Episódio de beber excessivo. ${ }^{5}$ Definido pelo WHO-Rose Angina Questionnaire.

${ }^{1}$ Adjusted for design. ${ }^{2}$ One missing. ${ }^{3}$ Alcohol volume in grams per day ${ }^{4} \mathrm{HED}=$ Heavy Episodic Drinking ${ }^{5}$ Defined by the WHO-Rose Angina Questionnaire.

que ingerem até 19g de álcool por dia, sem episódios de embriaguez, quando comparado aos demais, já fora relatado por outros estudos. Na verdade, um potencial efeito protetor do beber moderado tem sido parte do folclore do beber por séculos, tendo sido empiricamente demonstrado por Pearl por volta de $1920^{18}$. Na ciência moderna, a publicação de Klatsky et al. em 1974 iniciou uma discussão séria a respeito do efeito protetor do beber moderado para a doença coronariana ${ }^{19}$. Desde então, muitos pesquisadores examinaram este ponto, atribuindo o efeito protetor à inclusão dos ex-bebedores no grupo dos sujeitos abstinentes. Isto produziria um viés, na medida em que o número significativo de abstinentes era, na verdade, ex-bebedores que tinham parado com a bebida por motivos de saúde - o assim chamado efeito "sick-quitter" 20. Embora isto possa ter sido utilizado para compreender efeito protetor em outros estudos, no caso da presente pesquisa ele não se aplica, visto que foi possível separar os ex-bebedores dos abstinentes a vida toda. $\mathrm{O}$ achado sobre o efeito protetor do beber moderado (até 19g de álcool) comparado à abstinência a vida toda é consistente com outros estudos, como, por exemplo, o estudo de caso-controle de Piegas et al..$^{12}$, no 
Tabela 2 - Odds ratios e intervalos de confiança (95\%) para risco cardiaco ${ }^{1}$ e padrão de uso de álcool, ajustados para variáveis sociodemográficas, tabagismo e índice de massa corpórea em amostra da região metropolitana de São Paulo $(n=1.488)$.

Table 2 - Odds ratios (95\% confidence limits) for cardiac risk' and alcohol use, mutually adjusted for demographics variables, smoking and body mass indices in metropolitan Sao Paulo area $(n=1,485)$.

\begin{tabular}{|c|c|c|c|}
\hline & OR & $95 \% \mathrm{Cl}$ & P-value \\
\hline Idade (em anos) & 1,02 & $1,01-1,03$ & 0,001 \\
\hline \multicolumn{4}{|l|}{ Sexo } \\
\hline Homem & 1,00 & & \\
\hline Mulher & 1,78 & $1,12-2,83$ & 0,01 \\
\hline Uso de álcool ${ }^{2}$ & & & 0,01 \\
\hline 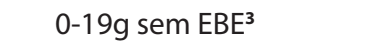 & 1,00 & & \\
\hline Abstinentes - vida toda & 2,23 & $1,27-3,90$ & 0,005 \\
\hline $0-19 \mathrm{~g}$ com EBE & 3,95 & $0,81-19,2$ & 0,09 \\
\hline $20 \mathrm{~g}$ ou mais & 1,43 & $0,50-4,07$ & 0,49 \\
\hline Ex-bebedores & 2,37 & $1,32-4,23$ & 0,004 \\
\hline Grupo étnico & & & 0,04 \\
\hline Branco & 1,00 & & \\
\hline Negro & 1,98 & $1,17-3,32$ & 0,01 \\
\hline Mulato & 1,02 & $0,65-1,62$ & 0,91 \\
\hline Outros & 0,76 & $0,23-2,56$ & 0,65 \\
\hline Tabagismo & & & 0,04 \\
\hline Nunca fumou & 1,00 & & \\
\hline Ex-fumante & 1,12 & $0,68-1,78$ & 0,69 \\
\hline Fumante & 1,93 & $1,15-3,04$ & 0,01 \\
\hline Índice de massa corpórea ${ }^{4}$ & 1,02 & $0,99-1,07$ & 0,16 \\
\hline
\end{tabular}

'Definido pelo WHO-Rose Angina Questionnaire. ${ }^{2} \mathrm{O}$ volume de álcool consumido é expresso em gramas por dia. ${ }^{\mathrm{B} E B E}=$ Episódio de beber excessivo. ${ }^{4}$ Índice de Massa corpóreo foi definido como peso $(\mathrm{em} \mathrm{kg}$ ) dividido pela altura em metros ao quadrado $\left(\mathrm{m}^{2}\right)$.

'Defined by the WHO-Rose Angina Questionnaire. ${ }^{2}$ Alcohol volume in grams per day ${ }^{3} \mathrm{HED}=$ Heavy Episodic Drinking ${ }^{4}$ Body mass index - defined as weight (in $\mathrm{kg}$ ) divided by height squared $\left(\mathrm{m}^{2}\right)$.

qual os autores observaram um efeito protetor do uso de álcool contra infarto do miocárdio. Infelizmente, no estudo de Piegas et al. ${ }^{12}$ não foi investigada a ocorrência de EBE, o que impossibilita avaliar se a proteção se mantinha na presença de episódios de embriaguez. Do mesmo modo, no presente estudo observou-se apenas uma tendência daqueles com ingestão ate $19 \mathrm{~g}$ e EBE terem perdido o efeito protetor. É possível que, com um número maior de sujeitos nesta condição, e consequentemente maior poder estatístico, o impacto do EBE diminuindo o efeito protetor fosse significativo. Mukamal et al. ${ }^{21}$ encontraram uma associação inversa entre doença coronariana e ingestão de 5,0 a 14,9g de álcool por dia, a partir de uma a mostra composta exclusivamente por sujeitos sãos, com estilo de vida saudável, em um estudo de coorte.
Da mesma maneira, o maior risco de doença coronariana entre ex-bebedores é também consistente com a literatura ${ }^{22}$. Graham $^{23}$ estudou razões para abstinências e características dos abstinentes entre adultos. A autora observou que, comparados com outros abstinentes, os ex-bebedores referiam significativamente diferentes razões para não beber, o que incluía preocupações com a saúde, problemas de saúde e uso de medicação.

A associação entre maior índice de massa corpóreo e tabagismo não surpreende, dado que são fatores de risco muito bem descritos para doença coronariana ${ }^{12}$. Por outro lado, o maior risco associado à etnia negra, embora não tão consensual quanto os fatores de risco anteriores, já foi observado em estudos anteriores. Fuchs et al. ${ }^{24}$, estudando a incidência de doença 
coronariana em comunidades dos EUA, encontrou uma forte associação entre a ocorrência desta doença e o consumo de álcool na faixa de 140-220g de etanol por semana, em todas as etnias, exceto entre brancos. Pletcher et al. ${ }^{25}$ examinaram a associação entre consumo de álcool e calcificação nas artérias coronárias, observando diferenças entre as etnias estudadas nas frequências destas calcificações nos bebedores pesados. De acordo com as conclusões dos autores, os achados sugerem a presença de efeitos pró-aterogênicos entre os afrodescendentes americanos. Um achado que também surpreende foi o maior risco entre as mulheres, que pode estar associado ao fato de as mulheres admitirem com maior facilidade a existência de sintomas físicos, quando comparadas aos homens, um aspecto fundamental quando o rastreamento se baseia em questionário autorrespondido.

O padrão de uso de álcool parece ser um importante preditor de doença coronariana. Em um estudo de metanálise, Bagnardo et al. observaram que EBE se associou a maior risco de doença coronariana, mesmo após controle para volume total de consumo de álcool ${ }^{26}$. No entanto, este estudo examinou apenas seis artigos, dado o pequeno número de estudos epidemiológicos que incluíram EBE como uma medida a ser investigada. Em outra metanálise ${ }^{27}$, que incluiu estudos de caso-controle e coortes, Roerrecke e Rehm (2010) observaram que o efeito protetor moderado do álcool desaparece quando o beber moderado é combinado com EBE mesmo que ocasionais. Ambas as metanálises fornecem informações importantes sobre esta associação; todavia, não incluem estudos realizados em países em desenvolvimento, dada a escassez de pesquisas que avaliem álcool e risco cardíaco nestas regiões.

Moraes et al. ${ }^{11}$ encontraram uma associação positiva entre maior risco de consumo de álcool e doença cardiovascular, mas, como já mencionado, esse estudo não investigou EBE. No presente estudo, EBE tendeu a se associar a maior risco cardíaco, mesmo entre os sujeitos que bebem até $19 \mathrm{~g}$ de álcool por dia. EBE já é uma preocupação em saúde publica no Brasil, devido à sua elevada prevalência. Lima et al. ${ }^{28}$ observaram uma prevalência de $35,8 \%$ de EBE entre residentes da região metropolitana de São Paulo. Guimarães et al. ${ }^{29}$ encontraram uma prevalência de $52,9 \%$ de consumo abusivo entre os homens e $26,8 \%$ entre as mulheres em diferentes regiões do Estado de São Paulo. Laranjeira et al..$^{30}$, em amostra nacional brasileira, observaram entre os bebedores uma prevalência de $28 \%$ de EBE no ano anterior à entrevista. Estes estudos, bem como outros conduzidos no país, reforçam que o EBE constitui um importante problema de saúde pública no Brasil.

Uma limitação presente em diversos estudos sobre álcool é que a informação sobre este consumo é frequentemente autorreferida, sendo assim possível algum grau de subestimação. Há apenas um estudo sobre confiabilidade de relato de uso de álcool em amostra brasileira ${ }^{31}$, no qual os autores encontraram coeficiente Kappa de 0,54 a 0,78 , com uma tendência a aumento entre os mais idosos e sujeitos com maior escolaridade. Outra possível limitação desta pesquisa está relacionada ao instrumento utilizado (WHO-RAQ), que se trata de um instrumento de rastreamento, sem sensibilidade e especificidade estabelecidas no Brasil, mas com boas propriedades psicométricas em outros locais ${ }^{15,16}$. Uma última limitação diz respeito ao pequeno número de sujeitos em algumas faixas de consumo de álcool, o que limita o poder do estudo.

A despeito das limitações, este estudo acrescenta informações sobre uso de álcool e risco cardíaco no país, um tema ainda insuficientemente estudado. Contribui ainda com a discussão sobre a importância de avaliar mais detalhadamente o uso de álcool quando da avaliação de risco cardíaco, tanto na prática clínica quanto em termos de pesquisa. Em relação à pratica clínica é importante ressaltar a impossibilidade de recomendar o uso de álcool como medida protetora ao risco cardíaco, pois além de seu uso mesmo moderado estar associado a outros desfechos, como o câncer de mama ${ }^{32}$, 
um número razoável de sujeitos pode evoluir para uso pesado, outro importante problema de saúde pública. Beber "pesado" é uma preocupação em termos de mortes violentas no país e estudos recentes têm mostrado um aumento significativo deste padrão de uso ${ }^{33}$. A potencial associação de uso abusivo de álcool com risco cardíaco enfatiza a necessidade de se conhecer o problema em detalhe e um alerta para a necessidade de políticas públicas efetivas de controle sobre o consumo de álcool no Brasil.

\section{Referências}

1. World Health Organization. Global Burden of Disease. Geneva; 2003.

2. Rehm J, Taylor B, Room R. Global Burden of disease from alcohol, illicit drugs and tobacco. Drug Alcohol Rev 2006; 25: 503-13.

3. Corrao G, Rubbiati L, Bagnardi V, Zambon A, Poikolainen $\mathrm{K}$. Alcohol and coronary heart disease: a meta-analysis. Addiction 2000; 95: 1505-23.

4. McKee M, Briton A. The positive relationship between alcohol and heart disease in Eastern Europe: potential physiological mechanisms. J Royal Soc Med 1998; 91: 402-7.

5. Puddey IB, Rakic V, Dimmitt SB, Beilin LJ. Influence of pattern of drinking on cardiovascular disease and cardiovascular risk factors - a review. Addiction 1999; 94: $649-63$.

6. Rehm J, Sempos CT, Trevisan M. Average volume of alcohol consumption, patterns of drinking and risk of coronary heart disease - a review. J Cardiov Risk 2003; 10: $15-20$.

7. Rehm J, Room R, Graham K, Monteiro M, Gmel G, Sempos CT. The relationship of average volume of alcohol consumption and patterns of drinking to burden of disease - an overview. Addiction 2003; 98: 1209-28.

8. Pappa E, Kontodimopoulos N, Papadopoulos AA, Pallikarona G, Niakas D, Tountas Y. Factors affecting use of preventive tests for cardiovascular risk among Greeks. Int J Environ Res Public Health 2009; 6: 2712-24.

9. Passos LCS, Lopes AA, Lessa I, Sanches A, Santos-Jesus R. Mortality attributed to myocardial infarction in the male and female population of Salvador, BA, between 1981 and 1996. Braz Arch Cardiol 2000; 74: 332-4.

10. Leeder S, Raymond S, Greenberg H, Liu H, Esson K. A race against time: the challenge of cardiovascular disease in developing economies. New York: Columbia University Press; 2004.

11. Moraes RS, Fuchs FD, Moreira LB, Wiehe M, Pereira GM, Fuchs SC. Risk factors for cardiovascular disease in a Brazilian population-based cohort study. Int J Cardiology 2002; 90: 205-11.
12. Piegas LS, Avezum A, Pereira JC, Rossi Neto JM, Hoepfner C, Farran JA, et al. Risk factors for myocardial infarction in Brazil. Am Heart J 2003; 146: 331-8.

13. Taylor B, Rehm J, Aburto JTC, Bejarano J, Cayetano C, Kerr-Corrêa F, et al. Alcohol, gender, culture and harms in the Americas: PAHO Multicentric Study final report. Washington: Pan American Health Organization; 2007.

14. Lawlor DA, Adamson J, Ebrahim S. Performance of the WHO Rose angina questionnaire in post-menopausal women: Are all of the questions necessary? J Epidemiol Community Health 2003; 57: 538-41.

15. Graff-Iversen S, Selmer R, Løchen ML. Rose angina predicts 23-year coronary heart disease mortality in women and men aged 40-49 years. Heart 2008; 94: 482-6.

16. Bodegard J, Erikssen G, Bjornholt JV, Thelle D, Erikssen J. Possible angina detected by the WHO angina questionnaire in apparently healthy men with a normal exercise ECG: coronary heart disease or not? A 26 year follow up study. Heart 2004; 90: 627-32.

17. Kleinbaum DG. Logistic Regression: A self-learning text. New York: Springer; 1994.

18. Pearl R. Alcohol and Longevity. New York: Alfred A. Knopf; 1926.

19. Klatsky AL, Friedman GD, Siegelaub AB. Alcohol consumption before myocardial infarction. Results from the Kaiser-Permanent epidemiologic study of myocardial infarction. Ann Intern Med 1974; 81: 294-301.

20. Shaper AG,Wannamethee G, Wlaker, M. Alcohol and mortality in British men: explaining the U-shaped curve. Lancet 1988; 2(8623): 1267-73.

21. Mukamal KJ, Chiuve SE, Rimm EB. Alcohol consumption and risk for coronary heart disease in men with healthy lifestyles. Arch Intern Med 2006; 166: 2145-50.

22. Shaper AG. Alcohol and mortality: a review of prospective studies. Br J Addict 1990; 85: 837-47.

23. Graham K. Alcohol abstention among older adults: reasons for abstaining and characteristics of abstainers. Addict Res 1998; 6: 473-87. 
24. Fuchs FD, Chambless LE, Folsom AR, Eigenbrodt ML, Duncan BB, Gilbert A et al. Association between alcoholic beverage consumption and incidence of coronary heart disease in whites and blacks. $A m \mathrm{~J}$ Epidemiol 2004; 160: 466-74.

25. Pletcher MJ, Varosy P, Kiefe CI, Lewis CE, Sidney S, Hulley SB. Alcohol consumption, binge drinking, and early coronary calcification: findings from the coronary artery risk development in young adults (CARDIA) study. Am J Epidemiol 2005; 161: 423-33.

26. Bagnardi V, Zatonski W, Scotti L, La Vecchia C, Corrao G. Does drinking pattern modify the effect of alcohol on the risk of coronary heart disease? Evidence from a meta-analysis. J Epidemiol Community Health 2008; 62 : 615-9.

27. Roerrecke M, Rehm J. Irreegular heavy drinking occasions and risk of ischemic heart disease: A systematic review and meta-analysis. Am J Epidemiol 2010; 171: 633-44.

28. Lima MCP, Kerr-Corrêa F, Tucci AM, Simão MO, Oliveira JB Cavariani MB. Gender difference in heavy alcohol use: a general population survey (The Genacis Project) of São Paulo City, Brazil. Contemp Drug Problems 2007; 34: 427-44.
29. Guimarães VV, Florindo AA, Stopa AR, César CLG, Barros MBA, Carandina L et al. Consumo abusivo e dependência de álcool em população adulta no estado de São Paulo, Brasil. Rev Bras Epidemiol 2010; 13: 314-25.

30. Laranjeira R, Pinsky I, Sanches M, Zaleski M, Caetano R. Alcohol use patterns among Brazilian adults. Rev Bras Psiquiatr 2010; 32: 231-41.

31. Chor D, Faerstein E, Alves MGM, Lopes CS. How reproducible is self-reported information on exposure to smoking, drinking, and dietary patterns? Evidence among Brazilian adults in the Pro-saúde Study. São Paulo Med J 2003; 12: 63-6.

32. Mørch LS, Johansen D, Thygesen LC, Tjønneland A, Løkkegaard E, Stahlberg C et al. Alcohol drinking, consumption patterns and breast cancer among Danish nurses: a cohort study. Eur J Pub Health 2007; 17(6): 6249 .

33. Moura EC, Malta DC. Consumo de bebidas alcoólicas na população adulta brasileira: características sociodemográficas e tendência. Rev Bras Epidemiol 2011; 14(S1): 61-70.

Recebido em: 16/01/12 Versão final apresentada em: 15/10/12 Aprovado em: $31 / 10 / 12$ 